\title{
Genetic Algorithm based Energy Efficient Data Gathering in Wireless Sensor Networks
}

\author{
T. Sujithra \\ Research Scholar \\ Department of Computer Science and Engineering \\ PSG College of Technology, Coimbatore, India
}

\author{
R. Venkatesan \\ Professor \\ Department of Computer Science and Engineering \\ PSG College of Technology, Coimbatore, India
}

\begin{abstract}
In this paper genetic algorithm based energy efficient data gathering approach is proposed to maximize the network lifetime in terms of rounds. The proposed approach has two phases, namely a setup phase and steady state phase. In the setup phase, the cluster formation is done based on the query sent by the base station in a dynamic fashion. The nodes, which satisfy the query are only allowed to participate in the clustering process others go to the sleep mode immediately. In which relay nodes are used as routing element, it collects the aggregated information from the cluster head and transmits to the base station via other relay node in a multihop fashion. It balances the network load among the relay nodes. It also reduces the packet loss because of data traffic. From the simulation results, we show that the proposed approach outperforms than the existing protocol in terms of increased network lifetime and decreased energy consumption.
\end{abstract}

\section{Keywords}

Multi-hop communication, Cluster centroid, Network lifetime, Cluster head, Relay node, Genetic algorithm.

\section{INTRODUCTION}

Sensors are used to measure physical parameters of the environment. It comprises of sensor and actuator, processing equipment, small size memory and transceiver [1]. In a wireless sensor network, as sensor nodes are smaller in size they are densely deployed to cover a large geographical area. For example forest fire monitoring, habitat monitoring, landslide monitoring, etc. Lifetime of the sensor node is the time at which the first sensor node in the network runs out of energy [2]. The lifetime of the wireless sensor network can be improved by various ways, such as efficient routing, effective clustering, cluster head election strategy and efficient sleep scheduling algorithm, etc. Sleep scheduling algorithm deals with the scheduling of the transceiver states [3]. The transceiver has three states, namely idle, transmit/receive and sleep state. In an idle mode, most of the parts in the transceiver are switched off and only significant parts of the transceiver are in active mode. It consumes only less amount of energy than the transmit/receive mode. In sleep mode, all the parts of the transceiver are switched off. Hence there is no energy loss at all. Meticulous scheduling of transceiver states will improve the lifetime of the sensor network. Energy efficiency plays a pivotal role in wireless sensor network as it affects the lifetime of the sensor network. Furthermore, once the sensor nodes are deployed, it is hard to recharge due to either the huge volume of sensor node deployment or human non-intervention characteristics of the deployment area. In a wireless sensor network, most of the energy is consumed for communication purpose. The amount of energy consumed in wireless communication is directly proportional to the communication distance. Therefore, the long distance communication is not preferred. Usually, the data collection in large geographical area is accomplished via multi-hop communication. Furthermore, the expenditure of energy in multi-hop communication is heterogeneous as nodes near the base station (BS) drain out very quickly. Because, these nodes are forced to forward the data of other nodes in the network to BS. In order to address the non-uniform consumption problem among the sensor nodes, we propose a genetic algorithm based energy efficient data gathering approach. The main idea is to

- Reduce the energy consumption by controlling the cluster count and its density based on the query sent by the BS.

- Improve the network lifetime by using relay nodes as routing element.

The rest of the paper is organized as follows i) Section 2 describes the related work of this paper ii) Section 3 discusses about network and energy consumption model iii) Section 4 discuss about the proposed protocol iv) Section 5 deals with the experimental results and discussion and v) Section 6 gives the conclusion.

\section{RELATED WORK}

This section discusses about the works that are under study leading to fresh estimates. Based on the sensor node deployment, wireless sensor network is classified into two, namely homogeneous network and heterogeneous network. Sensor nodes deployed in a homogeneous network having the identical communication capability and equal amount of energy, in which some of the nodes are selected as cluster head, because of energy insufficiency role of cluster head is rotated, however energy consumption is eventually distributed among the sensor nodes. Frequent cluster head re-election gives additional overhead [4] [5]. Heterogeneous network composes of small number of resource rich nodes and large number of energy limited basic sensor nodes. The basic sensor node has only limited communication capability and energy. Resource rich nodes are equipped with powerful transceivers and high battery. In which resource rich nodes are acting as cluster head and basic sensor nodes are acting as cluster members. However, cluster head re-election frequency is minimized by excessive energy. Placing resource rich nodes at appropriate location is difficult [6]. Event driven, query based, time driven and hybrid are possible ways of initiating the data transfer from sensors to the sink [7]. For any data gathering scheme, sensors should send their readings to the sink, it may be either static sink or mobile sink. Mostly in static sink. 
Approach data are transferred from sensors to the sink via multi-hop communication. Due to this, nodes near the static sink deplete its energy quickly. It makes the static sink unreachable. Clustering is a standard approach for achieving efficient and scalable performance in wireless sensor networks. Some of the clustering approaches are discussed as follows. Sangho Yi et al. [8] proposed power efficient and adaptive clustering hierarchy protocol for wireless sensor networks (PEACH) in 2007. The goal of this paper is to elect the cluster head $(\mathrm{CH})$ based on the characteristics of overhearing. It is an adaptive multi level clustering algorithm. During data transmission, packet is received by all the nodes in the cluster. But the node which is the owner of the packet will be elected as cluster head. Remaining nodes will become a cluster member, even they hear the packet. By this way, cluster head election is carried out. Heinzelman et al. [9] introduced centralized low adaptive clustering hierarchy (LEACH-C) in 2002. In this paper, BS acts as central coordinator. It takes the responsibility of cluster formation. Cluster head is elected based on the energy level of the sensor node. Node having maximum energy will get a higher chance to get elected as cluster head. The BS knows about the entire details about the network. Jau Yang Chang et al. [10] introduced an efficient cluster based power saving scheme for wireless sensor networks (SECA) in 2012. The main idea is to extend the network lifetime by using uniform cluster locations and balancing the network loading among the clusters. It partitions the entire network into equal sized clusters using KMeans algorithm. Cluster head is elected based on both hybrid energy level of the sensor node and distance between the sensor nodes. Smaragdakis $G$ et al. [11] presented a stable election protocol for clustered heterogeneous wireless sensor networks (SEP) in 2004. It handles the heterogeneous nodes in terms of energy. Cluster head is elected based on the residual energy of the nodes. Two kinds of nodes play an important role in the clustering process, namely advanced nodes and normal nodes. Nodes with highest energy are advanced nodes, whereas nodes with a nominal energy are normal nodes. Advanced node is having higher opportunity to get elected as cluster head than the normal node. Ye $\mathrm{M}$ et al. [12] presented an energy efficient clustering scheme in wireless sensor networks (EECS) in 2005. It is mainly designed for periodical data gathering application. Node having maximum residual energy is elected as cluster head in an autonomous manner. It uses distance based method to balance the load. Heinzelman et al. [4] introduced low energy adaptive clustering hierarchy (LEACH) in 2000. Clustering process is done in two phases, namely set up phase and steady state phase. Cluster formation and cluster head election is carried out in the set up phase. Data transmission is carried out in the steady state phase. Role of cluster head is rotated to balance the energy consumption. Steady state phase uses Time Division Multiple Access (TDMA) schedule for synchronizing the sensor nodes and cluster head which gives an effective data communication. During the cluster head election, the energy level of the node and distance between the $\mathrm{CH}$ and BS is not taken into account. Parul Bakaraniya et al. [13] proposed an improved version of the LEACH protocol for lifetime improvement in wireless sensor network (KLEACH) in 2013. The main idea is to find the center position of cluster by using K-Medoids clustering algorithm. A node which is closer to the center position of the cluster is elected as $\mathrm{CH}$. It avoids the problem of the unreachable node. Sasikumar et al. [14] presented K-Means clustering in wireless sensor networks (K-Means) in 2012. It focuses on finding centroids of the cluster using K-Means algorithm. The cluster head election process uses euclidean distance and hybrid energy level of the node as selection criteria. Node with minimum distance to the centroid and maximum energy is appointed as $\mathrm{CH}$. At the time of cluster head gets depleted, the node which is nearest to the depleted cluster head with maximum energy is elected as $\mathrm{CH}$.

\section{NETWORK AND ENERGY CONSUMPTION MODEL}

Genetic algorithm based energy efficient data gathering (GAEEDG) approach has two fold objective: reducing the energy consumption by controlling cluster count and its density, and increasing the network lifetime by using relay element based routing technique. The proposed approach is designed based on the following observations. WSN has three basic components: basic sensor nodes, resource rich nodes and base station. Some of their characteristics in WSN.

- Sensing environment composes of a large number of basic sensor nodes and limited number of resource rich nodes called relay node.

- The basic sensor node has only limited energy and communication capabilities.

- The basic sensor nodes are acting as data sources. It collects the information from the sensing environment.

- The resource rich node has a powerful transceiver and communication capabilities. It acts as routing element.

- The sensor node cannot be recharged after deployment.

- A Base station is stationary. It is fixed at the centre of the sensing environment.

- Location of the relay nodes is known.

- SECA [10] and LEACH [4] are taken as the benchmarking system.

\subsection{Network model}

In our scenario, consider PXQ sensing area comprises of a $\mathrm{BS}$, ' $\mathrm{n}$ ' number of sensor nodes that are randomly distributed over the sensing environment and ' $m$ ' number of relay nodes is eventually distributed at the regular intervals. We assume that, BS is positioned at the centre of the sensing environment. The relay nodes are always to be in active mode. The nodes in the sensing environment are clustered based on the query sent by BS. The nodes who satisfy the query are only allowed to retain in the transmit mode and actively participating in the clustering process. The node, who does not satisfy the query go to the sleep state immediately. It is not allowed to participate in the clustering process. Cluster head collects the information from its cluster members. Relay node collects the information from its immediate neighborhood $\mathrm{CH}$ and forwards it to BS via other relay node in a multi-hop fashion. The relay node acts as routing element. AODV algorithm is used to find the shortest path.

\subsection{Energy consumption model}

According to the energy consumption model [4] [10], the energy is needed for transmitting ' $M$ ' bits to a distance ' $d$ ' is expressed as follows. 


$$
E_{T x}(L, d)=E_{\text {elec }} \cdot L+\epsilon_{\text {amp }} \cdot L
$$

The energy is needed for receiving data is denoted as

$$
E_{R x}(L)=E_{\text {elec }} \cdot L
$$

Where $\mathrm{d}$ is the distance between the two sensor nodes, $\mathrm{E}_{\mathrm{TX}}(\mathrm{L}, \mathrm{d})$ is the transmitter energy consumption, and $\mathrm{E}_{\mathrm{Rx}}(\mathrm{L})$ is the receiver energy consumption. $\mathrm{E}_{\text {elec }}$ is the electronics energy consumption per bit in the transmitter and receiver sensor nodes. $\varepsilon_{\text {amp }}$ is the amplifier energy consumption. It is calculated by using

$\epsilon_{a m p}=\left\{\begin{array}{l}\epsilon_{f s} * d^{2}, \text { when } d \leq d_{0} \\ \epsilon_{m p} * d^{4}, \text { when } d>d_{0}\end{array}\right.$

Where $d_{0}$ is a threshold value. If the distance $d$ is less than $d_{0}$, the free space propagation model is used. Otherwise multipath propagation channel model is used. $\varepsilon_{\mathrm{fs}}, \varepsilon_{\mathrm{mp}}$ are communication energy parameters. Suppose that a non cluster head node $N$ transmits $\mathrm{L}_{\mathrm{N}}$ bits to the BS. Let $\mathrm{d}_{\mathrm{N}, \mathrm{CH}}$ be the distance between the non-cluster head node $\mathrm{N}$ and its cluster head node $\mathrm{CH}$. Let $\mathrm{d}_{\mathrm{CH}, \mathrm{RN}}$ be the distance between the cluster head and its immediate neighborhood relay node $R N$. Let $d_{R N, C H}$ be the distance between the relay node RN and the BS. Due to effective cluster head election multi-hop communication is reduced. Only the minimal amount of non- cluster head nodes are using multi-hop communication. Others using free space model for communicating with the $\mathrm{CH}$. The residual energy of the non-cluster head node $\left(\mathrm{CH}_{\text {non }}\right)$ is expressed as

$E_{\text {init }}-E_{T x}\left(L_{N}, d_{N}, C H\right)$

In addition, the residual energy of the cluster head node $(\mathrm{CH})$ is denoted as

$E_{\text {init }}-E_{R x}\left(L_{N}\right)-E_{D A}-E_{T x}\left(L_{N}, d_{C H, R N}\right)$

In order to reduce the energy hole problem in the static sink approach, Cluster head transmits its data to its immediate neighborhood relay node instead of using other sensor nodes in the sensing environment. The residual energy of the relay node (RN) is expressed as

$E_{\text {init }}-E_{R x}\left(L_{N}\right)-E_{T x}\left(L_{N}, d_{R N, B S}\right)$

The relay node acting as routing element, it collects the aggregated information from its immediate neighborhood $\mathrm{CH}$ and transfers it to the BS via other relay node.

\section{PROPOSED PROTOCOL}

In this algorithm, the operation includes two phases, setup and steady state phase.

\subsection{Setup Phase}

The main goal of this phase is to create clusters and find cluster heads. During the setup phase, the BS sends the query and collects the information about the position and energy level of the sensor nodes from only the nodes which satisfy the query.

\subsubsection{Dynamic Cluster Formation}

In existing approaches [4] [8] [9] [10] [11] [12], all the nodes In the sensing environment are taken into account at the time cluster formation. It results in an increased cluster count, cluster density, data traffic, energy consumption and burden of the aggregation process. In the proposed approach, states of the transceiver are scheduled based on the query sent by the BS. A query is a requirement that is required to measure from the environment. The BS transmits its query to all of its sensor nodes during its allotted time slot. The node which satisfying the query is only allowed to remain in the transmit mode and allowed to participate in the clustering process. Others go to the sleep mode immediately. Thus transceiver scheduling increases the network lifetime. Because the energy consumption in sleep mode is less compared to the energy consumption in the transmit/receive mode. It also reduces the count of the cluster formed.

\subsubsection{Cluster Centroid}

$\mathrm{K}$-Means algorithm is one of the prominent algorithms used to find the centroid of the cluster [14]. The centroid of the cluster $(\lambda)$ is calculated by using

$$
\lambda=\left(\frac{1}{n}\right) \times \sum\left(\min _{j} d^{2}\left(X_{i}, m_{j}\right)\right)
$$

Where $d\left(X_{i}, m_{j}\right)$ denotes Euclidean distance between the $X_{\mathrm{i}}$ and $\mathrm{m}_{\mathrm{j}} . \mathrm{m}_{j}$ denotes the mean value of the particular cluster and $\mathrm{X}_{\mathrm{i}}$ denotes the position of the nodes in that cluster.

\subsubsection{Cluster Head Election Strategy}

Cluster head election plays a vital role in conserving the energy level of the sensor node. Because poor cluster head election needs unnecessary re-clustering. It affects the lifetime of the sensor network. The position of the cluster head is a very important factor as long distance communication consumes more energy than the short distance communication. We consider both location and the energy level of the sensor node as criteria for electing a cluster head. Nodes that are closure to the cluster centroid and having a maximum energy level is elected as cluster head. It enables the cluster head to reach all of its cluster members with minimum energy consumption. It avoids multi-hop communication and reduces cluster head reelection frequency.

\subsubsection{Positioning of Base Station}

Proper positioning of base station also increases the lifetime of the sensor network. It enables the BS to reach all of its sensor nodes with minimum energy consumption. In our paper, the BS is placed at the centre of the sensing area. It increases the network lifetime. Center position $\left(\mathrm{x}_{\mathrm{c}}, \mathrm{y}_{\mathrm{c}}\right)$ of the sensing area is calculated by using

$\left(x_{c}, y_{c}\right)=\left(x_{i}+x_{j}\right) / 2,\left(y_{i}+y_{j}\right) / 2$

Where $\left(\mathrm{x}_{\mathrm{i}}, \mathrm{y}_{\mathrm{i}}\right)$ and $\left(\mathrm{x}_{\mathrm{j}}, \mathrm{y}_{\mathrm{j}}\right)$ are diagonal coordinates of the sensing area.

\subsection{Steady State Phase}

Once the clusters are created and the TDMA schedule is fixed, data transmission can begin. The cluster members send data to $\mathrm{CH}$ during their allocated transmission time. When all the data have been received, the cluster head performs aggregation and produce a single signal. It reduces the amount of information transmitted to the BS. Cluster head is always in an active mode. It delivers the aggregated data to either directly to BS or via relay node based on the cluster head classification. It is discussed as follows.

\subsubsection{Efficient Cluster Head Classification}

In order to simplify the data gathering process, our algorithm classifies the cluster head based on the Euclidean distance between the cluster head and the BS into two namely, first 
level cluster head and second level cluster head. If the BS is in the communication range of cluster head is taken as first level cluster head.otherwise, it is taken as the second level cluster head. It is represented as

$C=\left\{c_{1}, c_{2}, c_{3}, \ldots . ., c_{n}\right\}$

$D=\left\{d_{1}, d_{2}, d_{3}, \ldots . ., d_{n}\right\}$

Where $c_{1}, c_{2}, c_{3}, \ldots . ., c_{p}$ denotes cluster heads in the sensing environment. $d_{1}, d_{2}, d_{3}, \ldots . ., d_{p}$ refers distance between the cluster head and the BS.

$f_{c}=\left\{c_{i}: d\left(c_{i}, B S\right) \in \gamma, \exists C\right\}, 1 \geq i \leq n$

$s_{c}=\left\{c_{i}: d\left(c_{i}, B S\right) \notin \gamma, \exists C\right\}, 1 \geq i \leq n$

$f_{c}$ and $s_{c}$ denotes the first level and second level cluster heads. $\gamma$ is the maximum communication range of the cluster head. $\mathrm{d}\left(\mathrm{c}_{\mathrm{i}}, \mathrm{BS}\right)$ denotes the distance from the cluster head to the base station.

\subsubsection{Intra cluster communication}

In our approach data is gathered from the cluster head based on the cluster head classification. First level cluster head transmits its information to BS directly because of its closeness and Second level cluster transmits its data to the BS with the help of relay node. It reduces the energy hole problem in the static sink approach. AODV Algorithm is used to find the shortest path.

\subsection{Problem Representation}

In the proposed approach, genetic algorithm (GA) is used to minimize the count of the cluster formed and maximize the network lifetime in terms of rounds. GA begins with a population of possible solutions. Population is a predefined number of arbitrarily generated solutions. Each solution is represented through chromosome. Then GA evaluates the fitness of each solution based on the objective function. After that selection method choose the individuals from the population based on their fitness value for mating. Finally, genetic operators such as mutation and crossover are applied to produce a new generation.

\subsection{Dynamic Clustering with Genetic Algorithm}

Clustering is a one of the conventional approach for achieving efficient and scalable performance. The clustering process is considered to be NP- hard problem. Genetic algorithms have been successfully applied to a variety of NP-hard problems. We propose to apply GA to the problem of finding the optimum number of clusters based on minimizing the count of a cluster needs to effectively maximize the network lifetime. The elements in genetic algorithm are discussed in detail.

\subsubsection{Chromosome and gene}

A Chromosome is a group of genes. The representation of the network is called a chromosome. It has fixed sized length, which is determined by the number of nodes in the network. A gene is a bit string of random length. A gene index represents a node id and the gene value represents the sensing value.
Table 1. Chromosome examples

\begin{tabular}{|c|c|c|c|c|c|c|c|c|c|c|}
\hline Node ID & $\mathbf{1}$ & $\mathbf{2}$ & $\mathbf{3}$ & $\cdot$ &. &. &. &. & $\mathbf{9 9}$ & $\mathbf{1 0 0}$ \\
\hline $\begin{array}{c}\text { Sensing } \\
\text { value }\end{array}$ & 45 & 24 & 26 &. &. &. &. &. & 22 & 20 \\
\hline
\end{tabular}

The chromosome is a representation of a network of 100 sensor nodes. Even gene value is denoted as decimal, it can be encoded in binary format. For example, the chromosome given in fig 1 . can be represented as 101101, 011000, $011010, \ldots \ldots . ., 010110,010100$.

\subsubsection{Population}

A population is a group of individuals called chromosome, which represents the complete solution to a defined problem. The initial population is a group of randomly generated individuals. The various methods used in generating population are steady state GA, generational GA and elitism. In steady state GA, one or more individuals of the population are replaced. In generational GA, the entire population is replaced at each generation. In elitism, best chromosome is retained for future generation. In the proposed approach, elitism method is used for population generation.

\subsubsection{Population size}

The size of the population depends on the nature of the problem. In general, random initialization of the population is carried. The size of the population remains same for all generations.

\subsubsection{Fitness}

According to Kreinovich et al., 1933, fitness is the ability to pass on its genetic material to the next generation. In GA, fitness is evaluated using the objective function to solve a problem.

Objective function 1:

The aim is to minimize the cluster count by clustering the sensor nodes based on the objective function.

$f(x)=\operatorname{Max}\left(d_{i}\right)$

$d_{i}=x_{i}-q_{v}$

where $x_{i}$ denotes the sensing value of the node $i$ and $q_{v}$ denotes the query value broadcast by the BS. The individual has maximum fitness value is selected for clustering and others are excluded from the clustering process.

Objective function 2 :

The aim is to maximize the network lifetime by the effective scheduling of the transceiver states of the sensor node. This is given by the minimum of

$\sum E_{\text {cons }}(\mathrm{CH})+\sum E_{\text {cons }}\left(\mathrm{CH}_{\text {non }}\right)+\sum E_{\text {cons }}(R N)$

Where $\mathrm{E}_{\text {cons }}$ denotes the energy consumption.

\subsubsection{Selection}

In this phase, individuals are selected for reproduction. The chromosome, which has highest fitness value is getting higher probability of being selected. Widely used selection methods are roulette wheel selection, tournament selection and random selection. In our approach, roulette wheel selection method is used. The metric used in roulette wheel selection are

$\operatorname{Prob}_{i}=\frac{f\left(x_{i}\right)}{\sum f(x)}$ 
Expected count $=\frac{f\left(x_{i}\right)}{\frac{\sum f(x)}{n}}$

actual count $=$ roundoff $($ expected count $)$

\subsubsection{Reproduction}

Genetic operators such as mutation and crossover are used to produce fresh chromosomes.

\subsubsection{Crossover}

Crossover is also known as recombination. It is a genetic operator applies on a pair of chromosomes that generates two new chromosomes. Various crossover operations are used such as single point crossover, n-point crossover, and uniform crossover. In the proposed approach, single point crossover is used.

Table 2. Roulette wheel selection example

\begin{tabular}{|c|c|c|c|c|c|c|}
\hline $\begin{array}{c}\text { String } \\
\text { no }\end{array}$ & $\begin{array}{c}\text { Initial } \\
\text { population }\end{array}$ & $\begin{array}{c}\text { Sensing } \\
\text { value }\end{array}$ & $\begin{array}{l}\text { Fitness } \\
\mathbf{f}(\mathbf{x})= \\
\mathrm{x}_{\mathrm{i}}-\mathbf{q}_{\mathrm{v}} \\
\mathbf{q}_{\mathrm{v}}=\mathbf{2 0}\end{array}$ & $\operatorname{Prob}_{i}$ & $\begin{array}{c}\text { Expected } \\
\text { count }\end{array}$ & $\begin{array}{l}\text { Actual } \\
\text { count }\end{array}$ \\
\hline 1 & 010100 & 20 & 0 & 0 & 0 & 0 \\
\hline 2 & 010110 & 22 & 2 & 0.09 & 0.36 & 0 \\
\hline 3 & 011001 & 25 & 5 & 0.23 & 0.91 & 1 \\
\hline 4 & 100011 & 35 & 15 & 0.68 & 2.73 & 3 \\
\hline \multicolumn{3}{|c|}{ Sum } & 22 & 1.00 & 4 & 4 \\
\hline \multicolumn{3}{|c|}{ Average } & 5.5 & 0.25 & 1 & 1 \\
\hline \multicolumn{3}{|c|}{ Max } & 15 & 0.68 & 0.91 & 3 \\
\hline
\end{tabular}

Table 3. Single point crossover example

\begin{tabular}{|c|c|c|c|c|c|}
\hline $\begin{array}{c}\text { String } \\
\text { no }\end{array}$ & $\begin{array}{c}\text { Mating } \\
\text { Pool }\end{array}$ & $\begin{array}{c}\text { Cross } \\
\text { over } \\
\text { point }\end{array}$ & $\begin{array}{c}\text { Offspring } \\
\text { after } \\
\text { cross over }\end{array}$ & $\begin{array}{c}\mathbf{x} \\
\text { value }\end{array}$ & $\begin{array}{c}\text { Fitness } \\
\mathbf{f}(\mathbf{x})=\mathbf{x}_{\mathbf{i}}- \\
\mathbf{q}_{\mathbf{v}}\end{array}$ \\
\hline 1 & $100 \mid 011$ & 3 & 100001 & 33 & 13 \\
\hline 2 & $011 \mid 001$ & 3 & 011011 & 27 & 17 \\
\hline 3 & $1 \mid 00011$ & 1 & 100011 & 35 & 15 \\
\hline 4 & $1 \mid 00011$ & 1 & 100011 & 35 & 15 \\
\hline \multicolumn{5}{|c|}{ Sum } & 60 \\
\hline \multicolumn{5}{|c|}{ Average } & 15 \\
\hline
\end{tabular}

\subsubsection{Mutation}

Crossover can combine genetic information from two parents. But mutation only can introduce new genetic information in the new chromosome. In the proposed approach bitwise mutation is used.
Table 4. Bitwise mutation example

\begin{tabular}{|c|c|c|c|c|}
\hline $\begin{array}{c}\text { String } \\
\text { no }\end{array}$ & $\begin{array}{l}\text { Offspring } \\
\text { after cross } \\
\text { over }\end{array}$ & $\begin{array}{c}\text { Offspring } \\
\text { after } \\
\text { mutation }\end{array}$ & $\begin{array}{c}x \\
\text { value }\end{array}$ & $\begin{array}{c}\text { Fitness } \\
\mathbf{f}(\mathbf{x})=\mathbf{x}_{\mathrm{i}}-\mathbf{q}_{\mathrm{v}}\end{array}$ \\
\hline 1 & 100001 & 100001 & 33 & 13 \\
\hline 2 & 011011 & 011011 & 27 & 2 \\
\hline 3 & 100011 & 110011 & 51 & 31 \\
\hline 4 & 100011 & 100010 & 34 & 14 \\
\hline \multicolumn{4}{|c|}{ Sum } & 60 \\
\hline \multicolumn{4}{|c|}{ Average } & 15 \\
\hline \multicolumn{4}{|c|}{ Max } & 31 \\
\hline
\end{tabular}

The mutation rate varies between $\frac{1}{\text { chromosome length }}$

$\frac{1}{\text { population size }}$ and

\subsubsection{Termination}

This above process is repeated until the optimum number of clusters is formed.

\subsubsection{Algorithm}

Step 1: Initialize the population with predefined number of randomly generated individuals.

Step 2: Evaluate the population based on the fitness value of the objective function.

Step 3: Apply roulette wheel selection method to select the best individuals for mating.

Step 4: Apply the single point crossover on the pair of individuals and evaluate their fitness.

Step 5: Apply bitwise mutation and evaluate their fitness.

Step 6: Repeat the above steps until the optimum number of clusters is formed.

\section{EXPERIMENTAL RESULTS}

In this section, we evaluate the performance of our proposed genetic algorithm based energy efficient approach using the simulation model. We design a simulation environment by using $\mathrm{C}++$. The assumption of our simulation study is as follows.

Table - Simulation Parameters

\begin{tabular}{|c|c|}
\hline Number of nodes & 50,100 \\
\hline Node deployment & Random \\
\hline Mac & 802.11 \\
\hline Total sensing area & $100 \times 100 \mathrm{~m}$ \\
\hline Position of BS (X,Y) & $(50,50)$ \\
\hline Initial Energy & $2 \mathrm{~J}$ \\
\hline Energy needed for aggregation (EDA) & $5 \mathrm{~nJ} / \mathrm{bit} / \mathrm{signal}$ \\
\hline
\end{tabular}




\begin{tabular}{|c|c|}
\hline Electronics Energy (Eelec ) & $50 \mathrm{~nJ} / \mathrm{bit} /$ signal \\
\hline Number of Generation & $10-60$ \\
\hline Mutation Rate & 0.1 \\
\hline
\end{tabular}

\subsection{Performance Metrics}

1) Round: Round is defined as the receiving the data from all sensor nodes to the base station.

2) Total energy: It is defined as the sum of residual energy at all sensor nodes.

\subsection{Results Discussion}

Fig. 5.a shows the total network energy when number of sensor nodes are 50 and the sensing area is $100 \times 100 \mathrm{~m}$. It is very obvious that the total network energy of our proposed algorithm is higher than that of LEACH and SECA. The reason is, In the proposed approach, nodes who satisfy the query are only allowed to participate in the clustering process. Others go to the sleep mode immediately. It reduces the cluster count and its density. Thus, the energy consumption is reduced.

Fig 5.b, 5.c shows the first sensor node dead and half of the sensor nodes alive for three methods, when the number of sensor nodes is 50 and the sensing area is $100 \mathrm{X} 100 \mathrm{~m}$. It is evident that the lifetime of the sensor node of the proposed approach is higher than that of LEACH, and SECA. The reason is, in existing approaches, cluster head forwards its data via other sensor nodes in the sensing environment. It makes the nodes near the sink unreachable. In the proposed approach, cluster head forward its data to the base station via relay nodes. It results in increased network lifetime.

Fig 5.d plots the performance of benchmarking system and the proposed system as a function of rounds in terms of total network energy. From the Fig. 5.d we can see, when the density of the sensor nodes becomes larger, the advantage of the proposed system over benchmarking system becomes more because of dynamic clustering.

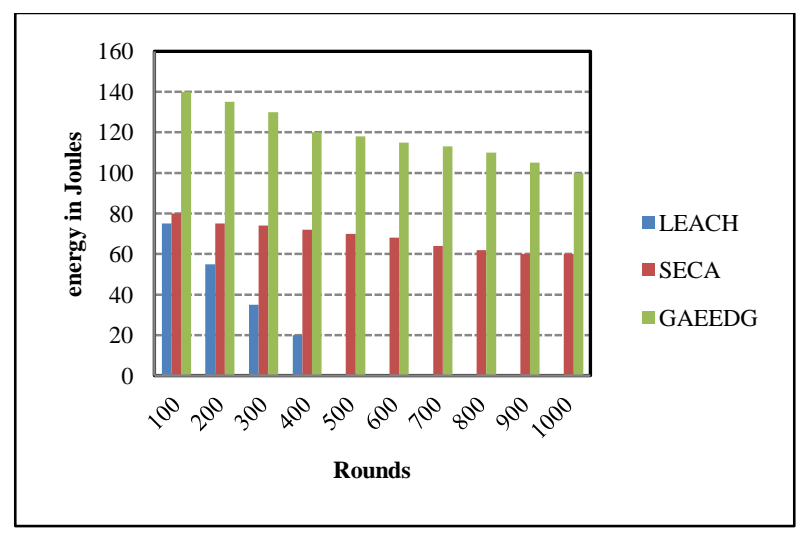

Figure 5.a Total network energy $($ Sensor nodes = 50)

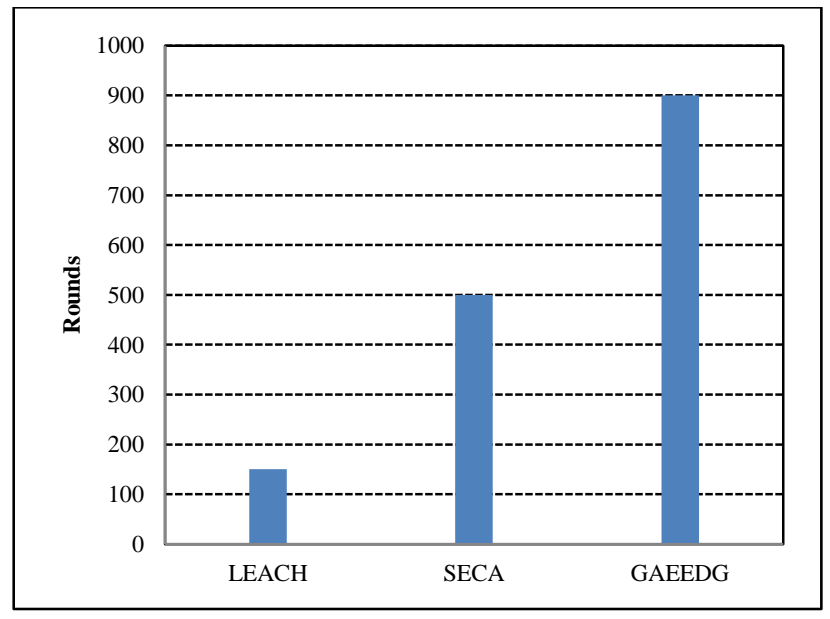

Figure 5.b First node dead $($ sensor node $=\mathbf{5 0})$

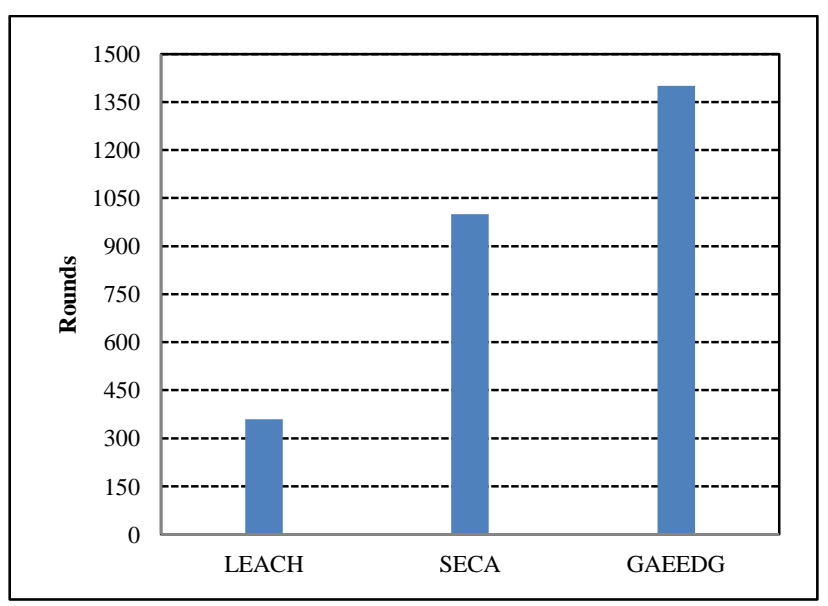

Figure 5.c Half of nodes alive (Sensor node $=\mathbf{5 0}$ )

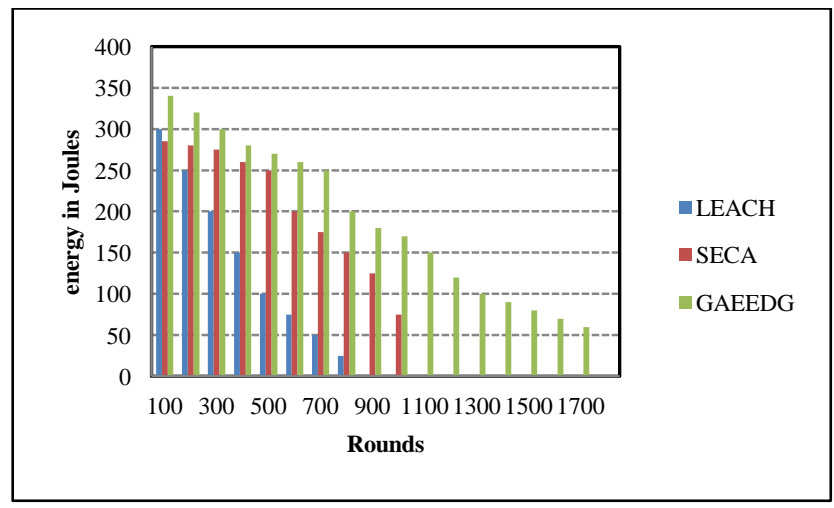

Figure 5.d Total network energy $($ Sensor nodes $=100)$

\section{CONCLUSION}

The proposed technique improves the network lifetime by controlling the nodes that are participating in the clustering process based on the query value. The simulation results infer that utilizing a GA based technique minimizes the total energy consumption. The proposed method begins with a predefined number of randomly generated chromosomes. Clustering is done based on the fitness value of the objective function. The simulation results demonstrate that the proposed GA based 
technique is an energy efficient approach to improve the lifetime of the system.

\section{REFERENCES}

[1] Jennifer Yick, Biswanath Mukherjee and Dipak Ghosal, "Wireless sensor Network Survey", In. Comput.Netw.,Elsevier., Vol.52, 2008, p.2292-2330.

[2] Kewei Sha and Weisong Shi, "Modeling the Lifetime of Wireless Sensor Networks ", In. Sensor Letters, American Scientific Publishers, Vol.3, 2005, p. 1-10.

[3] Beenu Baby and Joe Mathew Jacob, "A Survey on Sleep Schedule in Wireless Sensor Networks", In. International Journal of Engineering Research and Technology, Vol. 2, Issue 3, March 2013

[4] Heinzelman W.R, Chandrakasan A.P and Balakrishnan $\mathrm{H}$, "Energy-Efficient Communication Protocol for Wireless Microsensor Networks", In. Proceeding of 33rd Hawaii International Conference on System Sciences, Vol. 8, 2000, p.8020-8030.

[5] Liu X., Cao J., Lai S., Yang C., Wu H., and Xu Y., "Energy efficient clustering for WSN based structural health monitoring," In. Proc. IEEE INFOCOM, pp. 2768-2776, 2011.

[6] Zhang Z., Ma M., and Yang Y., "Energy efficient multihop polling in clusters of two-layered heterogeneous sensor networks," In. IEEE Transactions on Computer, pp. 231-245, 2008.

[7] Alsbou T.A.A, Hammoudeh M., Bandar Z, and Nisbet A., "An overview and classification of approaches to information extraction in wireless sensor networks," In. Proceedings of the 5th International Conference on Sensor Technologies and Applications (SENSORCOMM '11), pp. 255, 2011.

[8] Sangho Yi, Junyoung Heo, Yookun Cho and Jiman Hong, "PEACH : Power-Efficient and Adaptive
Clustering Hierarchy protocol for Wireless Sensor Networks, In IEEE parallel and distributed processing symposium(IPDPS), Florida, USA,2007.

[9] Wendi B. Heinzelman, Anantha P. Chandrakasan and Hari BalaKrishnan, "An Application-Specific Protocol Architecture for Wireless Microsensor Networks". In. IEEE transactions on wireless communications, Vol. 1, No. 4, October 2002.

[10] Jau-Yang Chang and Pei-Hao Ju," An efficient clusterbased power saving scheme for wireless sensor networks", In. Springer transaction on Wireless Communications and Networking, 2012.

[11] Smaragdakis G, Ibrahim Matta and Azer Bestavros, "SEP: A Stable Election Protocol for clustered heterogeneous wireless sensor networks", In. International conference on sensor and act or network protocols and applications SANPA, Boston, USA, 2004.

[12] Ye M, Li C, Chen G, Wu J and AlMYE, "EECS: An Energy Efficient Clustering Scheme in Wireless Sensor Networks", In. IEEE conference on performance computing, and communications (IPCCC), Phoenix Arizona, USA, 2005, p.535-40.

[13] Parul Bakaraniya and Sheetal Mehta, "K-LEACH: An Improved LEACH Protocol for Lifetime Improvement in WSN", In. International Journal of Engineering Trends and Technology, Vol.4, May 2013, p.1521-1526.

[14] Sasikumar P. and Sibaram Khara,"K-Means clustering in wireless sensor networks", In. IEEE Computer Society: Fourth International Conference on Computational Intelligence and Communication Networks, 2012.

[15] Kreinovich V., Quintana C., and Fuentes O., "Genetic algorithms- what fitness scaling is optimal," Cybernetics and Systems, Vol. 24, 1993, p. 9-26. 